\title{
ATENCIÓN INTERDISCIPLINAR PARA UNA ADECUADA ADHERENCIA AL TRATAMIENTO EN PACIENTES CON NEFRITIS LÚPICA
}

\section{INTERDISCIPLINARY CARE FOR ADEQUATE ADHERENCE TO TREATMENT IN PATIENTS WITH LUPUS NEPHRITIS}

\author{
TÍTULO CORTO: ATENCIÓN INTERDISCIPLINAR PARA UNA ADECUADA ADHERENCIA \\ AL TRATAMIENTO
}

Gladys Gaviria-García ${ }^{1}$, Gustavo Aroca-Martínez ${ }^{2}$, Gloria Lastre-Amell ${ }^{3}$, Carmen Sierra-Llamas ${ }^{4}$

Recibido en abril 29 de 2015

Aceptado en agosto 18 de 2015

\section{RESUMEN}

La revisión está fundamentada en el aporte en que cada disciplina debe brindar al paciente para un cuidado holístico, entre los cuales están la valoración médica, seguimiento y apoyo psicológico como soporte emocional, valoración y seguimiento nutricional como elemento fundamental en requerimientos primordiales, actividad física que optimice la calidad de vida, actividades sociales que logren incursionar al individuo en grupos activos, seguimiento por parte de enfermería al cumplimiento del tratamiento farmacológico ordenado, educación de auto cuidado y orientación a la familia. Lo novedoso de esta propuesta es básicamente llevar a cabo los cuidados del equipo interdisciplinario para la adherencia del tratamiento. Esta revisión permitió concluir, que los pacientes con Nefritis Lúpica (NL) tratados tras valoración y seguimiento holístico, como sistema de vigilancia y adherencia al tratamiento del cuidado integral, brindan mejor calidad de vida, minimiza los riesgos de complicación del paciente evitando hospitalizaciones recurrente.

Palabras clave: adherencia al tratamiento; nefritis lúpica; calidad de vida; atención al paciente; integralidad de la salud

\section{ABSTRACT}

The review is based on the contribution that each discipline should provide the patient for a holistic care, which include medical assessment, monitoring and counselling as emotional support, assessment and nutritional monitoring as a key element in core requirements, physical activity that optimize the quality of life, social activities that can enter the individual in active groups, follow-up by nurses to the fulfillment of the ordered drug treatment , car

1. Enfermera Magister en Educación. Universidad Simón Bolívar, Barranquilla - Colombia. Correo: ggaviria1@unisimonbolivar.edu.co

2. Médico Internista Nefrólogo. Universidad Simón Bolívar, Barranquilla - Colombia. Correo: garoca1@unisimonbolivar.edu.co

3. Enfermera Magister en Salud Pública. Universidad Simón Bolívar, Barranquilla - Colombia. Correo: glastre@unisimonbolivar.edu.co

4. Psicóloga Clínica y Magister en Psicología. Universidad Simón Bolívar, Barranquilla - Colombia. Correo: csierra@unisimonbolivar.edu.co 
care and orientation education to the family. The novelty of this proposal is to basically carry out care of the interdisciplinary team for treatment adherence. This review concluded that patients with lupus nephritis (NL) treated after assessment and follow-up holistic, such as system monitoring and adherence to the treatment of comprehensive care, provides better quality of life, and minimizes the risks of complication of the patient, avoiding recurrent hospitalizations.

Keywords: adherence; lupus nephritis; quality of life; patient care; comprehensiveness of health

\section{INTRODUCCIÓN}

L as enfermedades crónicas constituyen un problema de salud pública, se expresa que la incidencia y prevalencia han aumentado a nivel mundial, lo cual ha impactado en poblaciones vulnerable, coadyuvando al déficit de calidad de vida y altos indicadores de mortalidad, además de estos, también afecta la crisis económica, relaciones intrafamiliares y cambios de estilos de vida del paciente y su familia ${ }^{1}$. La Nefritis Lúpica (NL), aqueja a la mayoría de pacientes con Lupus eritematoso sistémico (LES) ${ }^{2,3}$, se presenta cuando los anticuerpos y complementos se acumulan causando inflamación y modificaciones de la función renal ${ }^{4,5}$. Una vez que se complica la enfermedad, se convierte en síndrome nefrítico y puede avanzar rápidamente hasta llegar a la enfermedad renal ${ }^{6}$, la cual suele desarrollarse a partir de los cinco años de edad luego de que se halla diagnosticado el lupus eritematoso (LES), por ello es considerada una enfermedad multisistémica crónica con gran connotación en su desarrollo, y manifestaciones clínicas ambigua del sistema inmunológico ${ }^{7}$,por lo tanto, se hace ineludible el abordaje temprano de tratamiento farmacológico y no farmacológico riguroso para prevenir deterioro precoz, que predominan la afección renal con todos los hallazgos sugestivos que comprometen a este órgano ${ }^{8}$.

Por consiguiente la NL como enfermedad crónica afecta al paciente desde varias perspectivas; la mayoría de pacientes son mujeres jóvenes en edades fértiles, de bajo estrato socio-económico. Por tanto, la detección temprana y un tratamiento apropiado a la luz de la integralidad pueden modificar cambios de estilos de vida que coadyuven al bienestar del paciente, familia y sistema de salud?.

Ahora bien, la Organización Mundial de la Salud ha abordado el tema de adherencia al tratamiento en pacientes con enfermedades crónicas no trasmisible (ECNT) como responsabilidad de salud pública, e incentiva al equipo de salud que mantenga una comunicación acorde con los pacientes, instruyendo sobre la importancia de controles para prevenir o tratar enfermedades como la $\mathrm{NL}^{10}$. Los determinantes psicológicos, sociales, económicos y culturales entre otros, son relevantes para una adecuada adherencia al tratamiento; además, se hace necesario desde el sistema de salud, establecer estrategias o modelos de atención que favorezcan la calidad de vida; y eviten enfermedades como la diabetes, que es un desorden metabólico pre disponente a la aparición de enfermedad o complicación renal ${ }^{11,12}$. Dentro de este marco, la educación es el elemento esencial para direccionar los pacientes; es por ello, que el equipo de salud debe estar articulado con este, como un sistema de vigilancia proporcionando así el cuidado en forma integral ${ }^{13,14}$, es importante señalar que la adherencia al tratamiento favorece la calidad de vida e impacta positivamente en indicadores de atención, disminuyendo los costos al sistema de salud ${ }^{15,16}$.

Es por ello que se hace necesario que los profesionales de la salud se planten interrogantes como, ¿qué importancia tiene brindar un cuidado integral como sistema de vigilancia a la adherencia del tratamiento a los pacientes con NL?, otro aspecto importante en esta revisión, es la articulación de la red de nefrología como sistema de vigilancia y seguimiento del aporte multidisciplinarios, el cual es trascendental para mejorar la adherencia y tratamiento en los pacientes con NL, desde el equipo interdisciplinario (médicos, psicólogos, enfermeras(os), fisioterapeutas y nutricionistas). Considerando que la adherencia se ha definido como el argumento en el cual el autocuidado de la persona coincide con las recomendaciones relacionadas con la salud e incluyen la capacidad del paciente para acudir a las valoraciones y seguimiento de las consultas programadas por el equipo interdisciplinario ${ }^{17}$. 
Lo anteriormente planteado, describe la importancia que tiene la intervención del equipo interdisciplinario para un manejo integral en los pacientes con NL, relacionado con la adherencia al tratamiento farmacológico y no farmacológico.

\section{Relevancia de la adherencia al tratamiento en pacientes con NL}

En este sentido, se comprende que la relevancia de la adherencia al tratamiento con NL, se fundamenta en aportes al mejoramiento de estilos de vida y prevención de riesgos de complicaciones del pacientes ${ }^{18-20}$, en efecto, la calidad de vida de pacientes con NL guarda relación con el manejo idóneo que brinde el equipo de salud, tal como, condiciones psicológicas, físicas, sociales, económicas, además, la vida de pareja y el contexto espiritual; los cuales, son condicionantes que debe reflexionar el médico para establecer tratamiento holístico, considerando que en su mayoría son individuos de poblaciones vulnerables ${ }^{15,21}$. Por lo tanto, la inadecuada prescripción médica del tratamiento podría inferir en la evolución de la enfermedad ${ }^{22}$. Si bien es cierto que los programas de promoción y prevención son estrategias del sistema de salud que emprenden lineamientos para mejorar estilos de vida de una población; de cumplirse estos programas en la sociedad estaríamos apuntando a reducir los riesgos de morbimortalidad por las complicaciones de la misma ${ }^{23}$, 24; teniendo en cuenta que ésta es una de las principales causas de muerte en países bajos y mayor costo en salud ${ }^{25}$. A continuación se explica la importancia del rol de cada uno de los profesionales en la adherencia al tratamiento en pacientes con NL.

\section{Apoyo médico en la adherencia al tratamiento en pacientes con NL}

La complicación más relevante de los pacientes con LES son las enfermedades renales, las cuales requieren de tratamiento sustitutivo, aproximadamente el $25 \%$ de los pacientes solicitan el apoyo médico; evidentemente, el médico juega un rol indispensable en el tratamiento, debido a su grado de responsabilidad en el diagnóstico de la enfermedad, por consiguiente, la biopsia renal es la herramienta más importante en el abordaje del diagnóstico sistémico para los nefrólogos, ya que de este, depende el tratamiento idóneo, además, la valoración y evaluación médica es necesaria como sistema de vigilancia permanente de la afección renal, la cual, deben realizarse por lo menos cada tres meses, al igual, que las pruebas de laboratorios, y la evaluación del tratamientos deben ser continuo, debido a que no existen definiciones estándares de patrones de respuesta al tratamientos ${ }^{26}$.

Ahora bien, el médico es el ordenador, que define el tratamiento e indica pautas de suministro ${ }^{27}$. Por consiguiente, su rol es contribuir al mejoramiento de la salud, ofreciendo conocimientos sobre la enfermedad, expidiendo exámenes, ordenando medicamentos y realizando evaluación y seguimiento; por esto, las manifestaciones clínicas con las que debuta esta enfermedad son importante discutirla entre el equipo interdisciplinario; considerando que el diagnóstico de LES es difícil, debido a diversas manifestaciones clínicas que presentan los pacientes, como en algunos casos potencialmente letales que fácilmente son confundidas con otros trastornos, lo cual constituye un reto clínico para un diagnóstico oportuno y certero por el equipo de salud $^{4-6,28,29}$; desde la perspectiva del inicio del tratamiento es importante resaltar que se si retrasa este, el paciente se expone a complicaciones pulmonares, sepsis, insuficiencia renal, en muchos casos irreversibles que llevan a la muerte; también debe señalarse que el desconocimiento de cuantificaciones serológicos de rutina y pruebas de laboratorio pueden inferir en decisiones del equipo de salud; por lo que se ha verificado en algunos estudios, los cuales describen el concepto unificado de características clínicas más frecuentes presentadas por pacientes con $\mathrm{NL}^{30-32}$.

Por consiguiente, se debe apuntar a evaluación periódica de adherencia al tratamiento farmacológico y no farmacológico, con el fin de abordar y confrontar lo ordenado por el medico a la luz de favorecer la calidad de vida del paciente ${ }^{33-35}$. En este orden de ideas, otro de los profesionales de la salud que se encarga de la atención directa y permanente con el paciente, es la enfermera, quien brinda los cuidados de forma holística especialmente en la adherencia al tratamiento.

\section{Rol de Enfermería en adherencia de tratamiento del paciente con NL}

Cabe señalar que la población con alta prevalencia de esta enfermedad son adultas jóvenes, de género femenino en etapa productiva, las cuales se ven afectadas desde el contexto fisiológico y psicológico que interfieren en su calidad de vida, de esta manera, el cuidado de enfermería se convierte en un importante atributo para brindar atención desde el aspecto humanízado ${ }^{36,38}$. La humanización y la eficacia de la atención en salud son aspectos que deben estar provistos para que enfermería 
brinde cuidados óptimos, tomando como principio ético la calidad de la atención, teniendo como aspecto fundamental la valoración del paciente, priorizando las necesidades, en la cual la enfermera pueda planificar, realizar y evaluar la atención, analizando e interpretando datos subjetivos y objetivos como resultado del proceso de atención de enfermería (PAE) ${ }^{39}$, brindando atención, eficiente y eficaz, desde el cuidado humanístico como lo narra Margarte Watson, quien describe el cuidado como elemento ético que coadyuve a satisfacer necesidades básica de individuo ${ }^{39,40}$. Un aspecto significativo de enfermería es el diagnostico según lo describe la NANDA $(1990)^{41}$, el cual es definido como un juicio crítico de respuestas humanas que da el individuo frente al problema de salud. Además, contribuye de manera perentoria en el cuidado; evalúa la responsabilidad y conducta del individuo frente a la adherencia al tratamiento, en el que incluye, la toma correcta del tratamiento farmacológico, dieta adecuada, realización de ejercicios y todas aquellas acciones que beneficien al individuo a mejorar su estilos de vida saludable ${ }^{42}$.

Así mismo, los pacientes con NL, conforman una población con elementos peculiares que los caracterizan entre el grupo de enfermedades crónicas no trasmisibles (ECNT), como son la severidad con la que avanza la enfermedad, el cambio permanente del estado de ánimo, y la apatía con la que la enfrenta ${ }^{38}$, por lo cual, la calidad de atención que se le brinde por parte del equipo de salud, debe ser oportuna, prevista de componentes éticos y humanísticos. Por ello, la enfermera cumple un rol destacado en el equipo ya que, está en mayor contacto con los pacientes; además, se puede decir que el cuidado de enfermería de manera holística, permite mejorar el estado de salud del paciente, reducir alteraciones propias de la enfermedad, minimizando los riesgos de complicaciones ${ }^{28}$. Asimismo, estudios revelan que los modelos de atención integral en salud, logran asertivamente resultados en el individuo, mejorando la calidad de vida del paciente, por lo tanto la propuesta de este estudio, se fundamentada en el aporte que cada disciplina debe brindar al paciente para una adherencia al tratamiento de manera integral con el fin de reducir complicaciones y mejorar las expectativas de vida del individuo ${ }^{12}$. Es por ello, que varios modelos teóricos han descrito como afrontar problemas, en aquellos que padecen NL, sin embargo, el trabajo del equipo de salud permite dimensionar elementos que sitúe a la enfermera a direccionar acciones de vigilancia y seguimiento del paciente por la cercanía y tiempo de contacto que mantiene con ellos, y el cual emplea para educar sobre auto cuidado, cambios de hábitos, promoción de la salud y prevención de enfermedad, al mismo tiempo que el cumplimiento del tratamiento farmacológico $27-29,35$.

En relación con el seguimiento y valoración del profesional de psicología, es importante destacar el proceso que emplea para fortalecer la adherencia al tratamiento, entre los cuales detallaremos seguidamente.

\section{Aporte del profesional de Psicología a pacientes con NL}

Uno de los aspectos relevantes en psicología de la salud es la evaluación no solo de la calidad de vida, estrategias de afrontamiento, creencias, estados emocionales, sino el de la adherencia al tratamiento. El término implica desde el interés de instrucciones recibidas por el personal médico, creencias positivas en su eficacia, unido a un papel activo en el proceso del tratamiento, desde la detección de la enfermedad hasta los controles médicos ${ }^{35}$. Asimismo, la salud mental, como eje integrador, es admitida como elemento primordial para el funcionamiento eficaz del ser humano, la cual reúne una serie de patrones emocionales, conductuales y cognitivos que favorecen al individuo en el afrontamiento de su realidad, haciéndolo consciente de sus propias fortalezas y limitaciones ${ }^{38}$.

En caso que en la evaluación se detecte que el paciente no se adhiera al tratamiento, el psicólogo, para lograrlo, utilizará técnicas para fortalecer la adherencia al tratamiento; para esto el terapeuta podrá utilizar la técnica del refuerzo, entre otras. Sin embargo, este modelo afirma que los efectos de refuerzo son limitados y difíciles de predecir (en algunos casos); de hecho, algunas investigaciones la consideran como un método que no da resultados útiles a la hora de mejorar el seguimiento de prescripciones médicas ${ }^{43}$, dependiendo de variables personales, sociales entre otras.

Continuando con otras de las técnicas utilizadas, la teoría cognitiva, basada en los principios de aprendizaje que subyacen a modelos conductuales, pero incluyen conceptos adicionales, como pensamientos automáticos, creencias irracionales acerca de su enfermedad, aporta técnicas como la reestructuración cognitiva la cual es una estrategia general de la terapia cognitiva comportamental, destinada a modificar el modo de interpretación y valoración subjetiva de la persona utilizando la práctica de hábitos cognitivos nuevos.

Por otra parte, la teoría de auto eficacia describe que es un concepto situacional específico que hace referencia 
a la confianza que depositan las personas en su propia capacidad para asumir comportamientos necesarios con el objetivo de obtener resultados deseados, específicos para una situación particular ${ }^{41}$. No obstante, la teoría de acción razonada sostiene que el determinante inmediato del comportamiento es la intensión del individuo para realizar conductas ${ }^{43}$.

Ahora bien, la intención del comportamiento, a su vez, es una función de actitudes de las personas hacia la conducta, determinada por creencias, de que el comportamiento produce resultados positivos o negativos y las normas subjetivas modeladas por la percepción acerca del valor que otras personas importantes otorgan a ese comportamiento; en este aspecto se tiene en cuenta la relación médico-paciente y a la vez las expectativas de vida que tiene el paciente.

Los factores que presentan una relación poco consistente con el grado de cumplimiento del tratamiento son: la edad, sexo, y rasgos de personalidad agregando la actitud y motivación que tiene el paciente para afrontar la enfermedad ${ }^{44}$ Investigadores descubrieron pocas diferencias en los niveles generales de cumplimiento entre hombres y mujeres. Las evidencias indican que las mujeres parecen seguir dietas alimenticias más sanas, incluyendo gran cantidad de verduras a la vez que obedecen en mayor medida a la hora de tomar fármacos ${ }^{44}$. El apoyo emocional es un factor que permite predecir cumplimiento en cierta medida, estudios afirman que el número de amigos pueden no estar relacionados con el nivel de compromiso del paciente, pero la calidad de las relaciones personales es un factor significativo que permite prever la adherencia ${ }^{43}$. Muchas personas no siguen el tratamiento por algunas razones como dificultad de afrontamiento a un nuevo estilo de vida, en especial en tratamientos que son prolongados, o una comunicación deficiente entre el terapeuta y el paciente ${ }^{38}$.

Ahora bien, las realidades acerca de la eficacia del cumplimento no están bien definidas; algunos estudios señalan que el cumplimiento compensa, mientras que otros sugieren lo contrario. De acuerdo a investigaciones realizadas ${ }^{38}$.se propone un programa eficaz para mejorar niveles de cumplimiento que contenga lo siguiente: explicar instrucciones con claridad, realizar prescripciones simples, llamada telefónica a los pacientes, posteriores a su citas incumplidas, diseñar recetas ajustadas a la rutina diaria de cada paciente, efectuar recompensa por un comportamiento disciplinado, recordatorio que permitan recordar hora de la toma, implicación del conyugue del paciente y el establecimiento de una red de apoyo.
Es por eso que el apoyo emocional contribuye al mejoramiento del paciente, puesto que este maneja una baja autoestima por su aspecto, por su enfermedad y por no llevar una vida normal. Por otro lado, se maneja temor por parte del paciente, el cual manifiesta miedo a morir, miedo a abandonar a su familia e incluso miedo a no haber vivido lo suficiente; la mayoría se sienten en desventaja ante los demás, pero son las mismas secuelas que deja la baja de autoestima que hace que se encuentren emociones y que se manifiesten los sentimientos ${ }^{43},{ }^{42}$. Otro aspecto, es la depresión que manifiesta cuando el médico da los resultados del diagnóstico de la enfermedad el cual, sino tiene un apoyo, el tiempo de recuperación de la situación al darle la noticia es lenta y es probable que no siga las prescripciones médicas.

Por tanto, el apoyo emocional juega un papel muy importante en el mejoramiento y la recuperación de los pacientes. En este caso es brindar apoyo a los pacientes con NL, ofreciéndoles que asistan a programas educativos sobre el manejo de su enfermedad, haciéndolos participes de su propia vida social y aportando iniciativas para que los pacientes no se aparten de su vida cotidiana ${ }^{43}$, ${ }^{41}$. Por otro lado, ofrece elementos que van a contribuir a la adherencia del tratamiento y participación activa por parte de la familia, promoviendo dinámica e integralidad al mismo ${ }^{46,47}$.

Es importante, que a los pacientes se le hagan procesos de intervención terapéutica, incluyendo a la familia, evitando que se realicen de manera aislada, puesto que la inclusión de la familia como acompañamiento, desde que se inicia, contribuye a que el paciente tenga un seguimiento continuo y permite mayor adhesión a la terapia, a través de una comunicación idónea del equipo terapéutico, familia y el entorno donde habita, siendo la respuesta terapéutica más efectiva con el equipo de salud $^{48,49}$. Por consiguiente, es importante y necesario el seguimiento a procesos de adhesión y adaptación al tratamiento, por parte del grupo interdisciplinario ${ }^{48,4}$. Otro profesional indispensable al equipo de trabajo es el nutricionista, el cual cumple un rol muy significativo con los pacientes con NL.

\section{Intervención del Nutricionista en la adherencia en pacientes con NL}

Con respecto a la nutrición, se considera el proceso espontáneo que el individuo suministra al organismo, el cual a través de ellos recibe sustancias químicas importante para la vida, y son llamadas desde el concepto de salud nutrientes esenciales e indispensable para el ser 
humano como fuente de energía y equilibrio metabólico, los cuales son suministrado a través de los alimentos; entre ellos tenemos; sales, carbohidratos, glucosa, aminoácidos, monosacáridos que para mejorar la calidad de vida y coadyuvan a la tolerancia de los tratamientos farmacológicos, por lo tanto, se hace necesario establecer una terapia efectiva para evitar proceso de malnutrición en los pacientes con NL que produzca descompensación al individuo ${ }^{50}$. No obstante, la intervención del profesional en nutrición no es nada facial, ya que tiene que enfrentarse a muchos aspectos del paciente como son los culturales, educativos, económico y sociales los cuales pueden intervenir en los hábitos alimentarios del individuo. Dicho de otra manera, la función del profesional de nutrición es de gran importancia para la injerencia de los grupos vulnerables con esta patología, instruir sobre adecuada alimentación, modificar estilos de alimentación "equívocos" es tarea indispensable, como soporte en la adherencia al tratamiento integral, el cual intenta apuntar a modelo saludable previniendo complicaciones de la enfermedad, la evaluación nutricional sobre la adherencia al tratamiento como medida terapéutica, indica al paciente los riesgos de una nutrición inadecuada a través de valoración antropométrica idónea, aportando a una adherencia al tratamiento de manera sistémica ${ }^{51,52}$. Como se ha dicho, el aporte de cada disciplina ofrece la oportunidad de una valoración y seguimiento holístico, el apoyo nutricional como sistema de vigilancia y adherencia al tratamiento del cuidado integral, brindan mejor calidad de vida, minimiza los riesgos de complicación del paciente evitando la hospitalización recurrente.

\section{Rol del fisioterapeuta en la adherencia al tratamiento en paciente con NL}

Los avances farmacológicos han sido de gran ayuda para el médico tratar ECNT, no obstante, no son la única solución para el tratamiento del paciente, a ellos se adicionan avances terapéuticos, en los cuales están; la terapia física, valoración nutricional, apoyo psicológico, educación de estilos de vida entre otros; como soporte al tratamiento farmacológico, aumentando expectativa de vida en estos pacientes y mejorando la calidad de vida ${ }^{53}$.

A pesar de estos avances se presentan inconvenientes que afectan a los profesionales de la salud, y es la falta de seguimiento de las prescripciones en los tratamientos. En el caso del fisioterapeuta esto se agudiza por el costo y el esfuerzo que le supone para el paciente el cumplir tales prescripciones. La mayoría de los pacientes en este campo sufren daños o molestias al realizar el tratamiento. A menudo, los profesionales de la salud prescriben las instrucciones en un lenguaje demasiado técnico que el paciente no deduce, de manera poco clara y muy rápidamente, puesto que el tiempo del que disponen para ver a los pacientes es breve. Además, no se cercioran que el paciente comprenda lo que se le pide y ni siquiera si es posible que lo haga. Si se le requiere al paciente que haga un determinado ejercicio y no dispone de algunos de los instrumentos necesarios para el mismo. Esta forma de trabajo asegura la no adherencia al tratamiento en este campo ${ }^{54}$.

Partiendo que el ejercicio es fundamental para un buen estado físico, por lo tanto el fisioterapeuta participa elaborando esquema de ejercicios que se adapte a la vida cotidiana del paciente, a sus horarios, estado físico y otras necesidades que lo hagan sentir activo, aportando desde esta disciplina al bienestar físico del paciente y a la adherencia al tratamiento terapéutic $0^{54}$ En los pacientes con NL los ejercicios ayudan a reducir rigidez y prevenir debilidad muscular de tal forma que los músculos desarrollen mayor capacidad para soportar las articulaciones dolorosas, incluso los ejercicios de estiramientos es tan eficaz como las actividades aeróbicas para mejorar su estado de ánimo sintiendo bienestar y disminuyendo la fatiga ${ }^{54}$. Hoy en día, el fisioterapeuta es consciente de la importancia de la adherencia al tratamiento y una de las estrategias que utiliza es la dedicación a educar al paciente proporcionando las instrucciones que debe seguir en el seguimiento de las actividades en casa.

\section{MATERIALES Y MÉTODOS}

La investigación con enfoque cualitativo, basado en el análisis documental específicamente de lupus eritematoso. Se hizo búsqueda en bases bibliográficas como Scielo, Elsevier, Pubmed, Latindex, Redalyc, Mediagraphics, Dialnet, utilizando descriptores de salud para la búsqueda como: enfermedades crónicas no trasmisibles, adherencia al tratamiento; Nefritis Lúpica; calidad de vida; atención al paciente; integralidad de la salud, cuidado integrales, salud pública. Para el desarrollo de pesquisa, se realizó en etapas de la siguiente forma. En la primera fase; recopilación de datos a través de exploración en base de datos incluyendo los de Nefro red del Caribe, se ejecutó durante el primer semestre del 2015 (enero a abril), artículos publicados entre los años 2005 y 2014, informes científicos de 2015, libros de 1953 hasta 1994, y normas de 2006 al 2007 escritos en español, inglés y portugués, identificando 70 estudios referentes a la temática; en una segunda fase, se clasificaron los estudios basados en categorías, los cuales se incluyeron 60 saberes con los que se adelantó 
la revisión; en la tercera fase, se realiza lectura y síntesis, procediendo así a la organización de esta, según categorías y finalmente 57 estudios aportaron al cumplimiento del objetivo. En la tabla 1 se muestra el proceso de revisión sistemática de investigaciones sobre la adherencia al tratamiento que se realizó para llevar a cabo el estudio.

Tabla 1. Revisión sistémica de investigaciones

\begin{tabular}{|c|c|c|c|}
\hline ARTícuLOS & $\mathbf{N}^{\circ}$ & BASES DE DATOS & $\mathbf{N}^{\circ}$ \\
\hline Artículos revisados & 70 & Scielo & 25 \\
\hline $\begin{array}{c}\text { Artículos } \\
\text { seleccionados }\end{array}$ & 51 & Elsevier & 4 \\
\hline Libros & 5 & Medigraphic & 1 \\
\hline $\begin{array}{c}\text { Normas y Leyes } \\
\text { eliminados no } \\
\text { elegibles. }\end{array}$ & 2 & Redalyc & 9 \\
\hline $\begin{array}{c}\text { Número de artículos } \\
\text { bibliográficos }\end{array}$ & $\mathbf{5 7}$ & Dialnet & 2 \\
\hline & & Pubmed & 1 \\
\hline & Latient Direct. & 1 \\
\hline Indexadas & Libros & 5 \\
\hline & & Normas & 2 \\
\hline
\end{tabular}

Fuente: Elaborado por autores

A pesar de los esfuerzos de la Organización Mundial de la Salud (OMS), las enfermedades crónicas no trasmisibles (ECNT), continúan siendo un problema de salud pública que ha conllevado al desgaste financiero del sistema de salud de los países, lo cual se ha visto reflejado en los altos perfiles epidemiológicos del mundo y América Latina. Estudios revelan, que 38 millones de personas que fallecen por ECNT, el $75 \%$ se da en países bajos ${ }^{55}$, lo que indica que los costos que genera son elevados, lo cual coadyuva al desgaste financiero, deterioro de calidad de vida, aumento de mortalidad, convirtiéndose en obstáculo para el desarrollo de un país ${ }^{56}$. Evidentemente, Colombia no está excepto de este flagelo, por tanto, se reconoce la importancia que tiene el estado frente a la problemática.

Dentro de este marco, investigaciones indican que el aumento de ECNT, está directamente relacionado con el tratamiento farmacológico y no farmacológico, además de otros condicionantes integradores que influyen en la calidad de vida del paciente como, dieta adecuada, manejo del estrés, actividades físicas, actividades sociales, entre otros cuidados que se complementan para la no la aparición de complicaciones de la enfermedad renal, o predisposición a la aparición de esta en pacientes con enfermedad como la hipertensión, la diabetes y el lupus eritematoso entre otros ${ }^{53}$.

Por otra parte, Bayes, citado por Vinaccia y Orozco ${ }^{57}$, describe que valorar la calidad de vida de pacientes crónicos es significativo porque a través de ello se puede saber el avance e impacto de la enfermedad y así mismo, contrastar la efectividad o no del tratamiento; evidentemente, esto permite identificar de forma adecuada efectos secundarios y adicionalmente mejorar las terapias. El proceso de evaluación de la adherencia permite al equipo de salud estar en permanente comunicación con el paciente y su familia, para valorar su evolución y seguimiento, sin duda este proceso es importante como sistema de vigilancia que favorece el autocuidado del paciente y la intervención del equipo, favoreciendo la calidad de vida del individuo, de igual manera impactando en indicadores de atención, disminución de costo al sistema de salud ${ }^{13,14}$.

Es conveniente anotar que en Colombia, la Ley 1122 de 2007, a través de políticas de salud pública, establece estrategias que pretende garantizar de forma integral, el cuidado de individuos y colectivos, las acciones van dirigidas a promover la participación de las personas de forma responsable sobre el auto cuidado, es así que la atención en salud, se encuentra regida por la política Nacional de Prestación de servicios de salud, y las instituciones de salud deben acatarlas para la atención del pacientes, cuyo objetivo final es garantizar el acceso y calidad de la atención del individuo; por lo tanto, los programas de promoción y prevención deben cumplir lo establecido en la norma a la luz de disminuir riesgos de 
complicaciones de las ECNT ${ }^{58}$. Dentro de esta perspectiva, esta revisión guarda relación con el estudio realizado en México, en el cual se efectuó una propuesta de programa enfocado al primer nivel de atención, el cual a través de la aplicación de guía de atención controlaría al pacientes diabéticos la glucemia, dislipidemia, albuminuria y presión arterial; este programa se aplicó como estrategia de prevención ofreciendo oportunidad de mejorar la atención que se daría a este grupo de pacientes previniendo a largo plazo la aparición de nefropatías diabéticas ${ }^{59}$.

Visto de esta forma, esta revisión busca expresar la importancia de brindar un cuidado integral como sistema de vigilancia a la adherencia del tratamiento a los pacientes con NL, trascendiendo en su calidad de vida y conservando así una comunicación permanente con el paciente y su familia, acoplada al equipo interdisciplinario (médicos, psicólogos, enfermeras(os), fisioterapeuta y nutricionista) y de esta forma se fortalecerían los programas de promoción y prevención.

Para finalizar este análisis, podemos decir que una adecuada intervención del equipo interdisciplinar como seguimiento a la adherencia del tratamiento en esta población, permite disminución de perfiles epidemiológicos, teniendo en cuenta que los estudios revelan el aumento de enfermedades renales que se avecina en las próximas décadas ${ }^{60}$.

\section{CONCLUSIÓN}

La presente revisión es relevante desde la promoción, prevención, tratamiento y rehabilitación en adherencia del tratamiento como cuidado integral a pacientes con NL, a partir de la perspectiva social, económica, psicológica, cultural, espiritual, además, pretende beneficiar al pacientes a través de la articulación de la red de nefrología como el sistema de vigilancia, seguimiento y aportes disciplinares que contribuirá a la orientación de cada profesional como calidad de vida con sus aportes de auto cuidado e impedir riesgos de complicaciones o muerte del pacientes con NL en la región Caribe colombiana.

\section{DECLARACIÓN SOBRE CONFLICTO DE INTERESES}

Los autores no reportan conflictos a declarar relacionados con la investigación.

\section{REFERENCIAS BIBLIOGRÁFICAS}

1. Trujano R, Vega Z, Navas C Saavedra Vázquez K. Interacción médico-paciente y su relación con el control del padecimiento en enfermos crónicos. Liberabit. 2011Dic; 17(2): 223-230.

2. Abud-Mendoza C. Nefritis lúpica: zavances en el conocimiento de su inmunopatogénesis sin los esperables logros terapéuticos? ReumatolClin. 2013 Mar; 9(2):77-79.

3. Ruiz G, Espinosa G, Frutos M, Jiménez J, Praga M, Pallares L. et al. Diagnóstico y tratamiento de la Nefritis Lúpica. Nefrología. Órgano Oficial de la Sociedad Española de Nefrología. 2012 Dic; 32(1): 1-35.

4. Estévez M., Chico A, Sánchez Y, Gutiérrez A, Chong A. Factores predictores de la respuesta a la terapia de inducción con ciclofosfamida en nefritis lúpica proliferativa. Cubanamed. 2007Sep; 46 (2):0-0.

5. Miranda-Hernández D, Cruz-Reyes C, Angeles U, Jara L, Angel Saavedra M. Predictores de respuesta al tratamiento en pacientes con nefritis lúpica. ReumatolClin. 2014 Ag; 10(3):164-169.

6. 6.Soyibo AK, Shah D, Barton EN, Williams W, Smith R. Renal histological findings in adults in Jamaica. West Indian Med J. 2009 Jun; 58 (3): 265-9.

7. Calvo J, Méndez A. Evolución de los pacientes con Nefritis Lúpica Proliferativa en el Servicio de Reumatología del Hospital San Juan de Dios que completaron tratamiento de 2 años hasta diciembre de 2010. Cl EMed UCR. 2013 Abr; 3 (5): 27-32.

8. González L, Vásquez G, Uribe O, Ramírez L: Nefropatía lúpica. Presentación clínica, clasificación y tratamiento. Colomb. Reumatol. 2006; 13(4): 307-333.

9. Braghetto I, Baronti P. Relación paciente-médico: Una alianza que fomenta la calidad. Chilena.2007 Oct; 59(5):385-392.

10. Holguín L, Correa D, Arrivillaga M, Cáceres D, Varela M. Adherencia al tratamiento de hipertensión arterial: efectividad de un programa de intervención biopsicosocial. Univ. Psychol. 2006 Dic; 5 (3): 535-547.

11. Quintana A, Merino J, Merino R, Cea J. Variables psicosociales asociadas a compensación metabólica de pacientes diabéticos de tipo 2. Méd Chile. 2008 Ago; 136(8): 1007-1014.

12. De la Rosa Y, Martín L, Bayarre H. Adherencia terapéutica y factores psicosociales en pacientes hipertensos. Cubana Med Gen Integr.2007 Jun; 23(1):89-99.

13. García-Milian A, Alonso Carbonel L, López Puig P, Yera Alós I, Ruiz Salvador A, Blanco Hernández $\mathrm{N}$. Reacciones adversas a medicamentos como causa de abandono del tratamiento farmacológico en hipertensos. Cubana Med Gen Integr. 2010; 25(1):1-10. 
14. Guerra V, Díaz A, Vidal K. La educación como estrategia para mejorar la adherencia de los pacientes en terapia dialítica. Cubana Enfermer. 2010; 26(2): 52-62.

15. Moix J, Casado M. Terapias Psicológicas para el Tratamiento del Dolor Crónico. Clínica y Salud. 2011Mar; 22 (1): 41-50.

16. Montes J, Casariego E, De Toro M, Mosquera E: La asistencia a pacientes crónicos y pluripatológicos. Magnitud e iniciativas para su manejo: La Declaración de Sevilla. Situación y propuestas en Galicia. Galicia Clin. 2012 Feb; 73 (1): 7-14.

17. Silva GE, Galeano E, Correa Jaime O. Adherencia al tratamiento Implicaciones de la no-adherencia. Acta Med Colomb. 2005 Dec; 30(4): 268-273.

18. Polanco N, Soto M, Rodríguez F. Presentación clínicopatológica de la nefropatía lúpica: experiencia de un centro mexicano. Colombo Reumatol. 2013 Abr; 20(2):80-90.

19. Builes C, Durango I, Velásquez C. Lupus eritematoso sistémico con anticuerpos antinucleares negativos y anemia hemolítica. Acta Médica Colombiana. 2010 Dic; 35 (4): 179-181.

20. Acosta P, Chaparro L, Rey C: Calidad de vida y estrategias de afrontamiento en pacientes con insuficiencia renal crónica sometidos a hemodiálisis, diálisis peritoneal o trasplante renal. Colombiana de Psicologia. 2008 Jul; (17): 9-26.

21. Vargas M, Pérez M. Dolor articular y lupus eritematoso sistémico. Un caso en imágenes. Acta Med Colomb. 2009 Mar; 34(1):1-50.

22. Ortega A, Moré C, Pérez L, Perez L, Rodriguez R, Lopez J. et al. Reactantes de fase aguda en pacientes hemodializados. Acta Médica del Centro. 2013 Jun; 7(3): 1-8.

23. Romaní F, Atencia F, Cuadra J, Canelo C. Lupus eritematoso sistémico, en un paciente varón: a propósito de un caso. An Fac med. 2008 Feb; 69(1): 37-41.

24. Córdova J, Lee G, Hernández M, Aguilar C, BarrigueteMeléndez J, Kuri Morales P. et al. Plan de Prevención y Tratamiento de las Enfermedades Crónicas: Sobrepeso, riesgo cardiovascular y diabetes mellitus, 2007-2012 y Sistema de Indicadores de Diabetes en México. Endocrinología y Nutrición 2008 Sep; 16 (3): 104-107.

25. Bonal R, Almenares H, Marzán M. Coaching de salud: un nuevo enfoque en el empoderamiento del paciente con enfermedades crónicas no transmisibles. Medisan. 2012 May; 16(5): 773-785.

26. Mogni G, Caputo M, Bottinelli Y. Remisiones y recaídas. Seguimiento a largo plazo de 84 pacientes. nefrología, diálisis y trasplante. 2014 Jun; 34(2):71-81.

27. Carrillo A, Laza C, Molina J. Estudio documental (20062013) sobre el auto cuidado en el día a día del paciente con enfermedad renal crónica. Enferm Nefrol. 2013 Sep; 16(3): 185-192.
28. Torralba M, Pérez I. La calidad de vida del paciente nefrológico desde la perspectiva bioética. Enferm Nefrol. 2011 Oct; 10(24):210-2015.

29. Diez-Canseco F, Ipince A, Toyama M, Benate-Galvez Y, Galán-Rodas E, Medina-Verástegui J. et al. Atendiendo la salud mental de las personas con enfermedades crónicas no transmisibles en el Perú: retos y oportunidades para la integración de cuidados en el primer nivel de atención. Perú Med Exp Salud Pública. 2014 Ene; 31(1):131-6.

30. Cardinalli A, Ginaca Al, Espada G, Pizzimenti M, Rivas, Leggire L, Griemberg G. Utilidad clínica de los anticuerpos anticitoplasma de neutrófilos en lupus eritematoso sistémico. Acta Bioquím Clín Latinoam. 2013 Mar; 47 (1): 145-53.

31. Treviño-Becerra, A. Tratamientos sustitutivos en enfermedad renal: diálisis peritoneal, hemodiálisis y trasplante renal. Cir Ciruj. 2009 Oct; 77 (5): 411-415.

32. Balparda J. Enfermedad renal crónica asociada a diacetil morfina (heroína). Iatreia. 2008 Dic; 21(4) 406-415.

33. Pellino M, Maiorana C, Lares M, Arana D, Arana D. Efecto cardíaco, inflamatorio y endotelial de pacientes trasplantados, con enfermedad renal crónica. Series de casos. Latinoamericana de Hipertensión. 2012 Sep; 7(3):48-52

34. Guerra V, Sanhueza O, Cáceres M. Calidad de vida de personas en hemodiálisis crónica: relación con variables socio-demográficas, médico-clínicas y de laboratorio. Latino-Am. Enfermagem. 2012 Oct; 20(5):838-46.

35. Villarroel P, Parra X, Ardiles L. Prevalencia y clasificación de enfermedad renal crónica en pacientes con diabetes mellitus tipo 2 en el centro comunitario de salud familiar Pantanosa, Frutillar. Méd Chile. 2012 Mar; 140(3): 287294.

36. Brannon L, Feist J. Psicología de la salud. España: Thomson Editores; 2001.

37. Massip C, Ortiz R, Llantá M, Peña M, Infante I. La evaluación de la satisfacción en salud: un reto a la calidad. Cubana Salud Pública. 2008 Jun; 34(4):0-0.

38. Vargas L. Marco para el cuidado de la salud en situaciones de enfermedad crónica. Investigación en Enfermería: Imagen y Desarrollo. 2010 Jun; 12 (1): 79-94.

39. McCormick K, Lang N, Zielstorff R, Milholland D, Saba V, Jacox A. Standard Classification Schemes for Nursing Language. Journal of the American Medical lnformatics Association. 1994 Dic; 1 (6):421-427.

40. González P, Chaves A. Proceso de atención de enfermería desde la perspectiva docente. Investigación en Enfermería. Imagen y Desarrollo. 2009 Dic; 11(2): 47-76

41. Skinner, Burrhus Frederic. Science and human behavior. New York: Macmillan: 1953.

42. Bandura A. Social Foundations of thought and action A social cognitive theory. Englewood Cliffs, Stanford, California: NJ Prentice- Hall; 1986. 
43. Ajzen I. Fishbein, M. Understanding attitudes and predicting social behavior. Englewood cliffs NJ: Prentice -Hall; 1980.

44. Seliwood W, Tarrrier N. Demografphic factor associated wiht extreme non-compliance in schizopherenia. Social Psychiatria and Psychiatric Epidemiology; 1994.

45. Fuente M, Arias M, Rosa A. Granulocitoaféresis: a propósito de tres casos Enfermería Nefrológica. Soc Esp Enferm Nefrol 2009 Jun; 12 (2): 161-163.

46. Rivas A, Vásquez M, Romero A, Escobar L, Sinning A, Calero Y. Adherencia farmacológica de la persona con enfermedad renal crónica en tratamiento de hemodiálisis. Duazary. 2012 Dic; 10(1):7-14

47. Vicente M, Olmedo San laureano, Jiménez A. Lupus eritematoso sistémico: A propósito de un caso clínico con presentación cutánea. Pediatr Aten Primaria 2013 Dic; 15(57): 55- 9.

48. Gorette M, Rolan M, Graça M. Aplicação da metodología da assistência a pacientes com Lúpus Eritematoso Sistêmico em pulsoterapia: uma experiência docente. Bras Enferm. 2007 Abr; 60(2): 229-32

49. González E, Ayala M, Ramírez M. Soporte nutricional a pacientes con enfermedad renal crónica dependientes de hemodiálisis. Medisan. 2009 Dic; 14(3):325-330

50. Holgado D, Maya I, Ramos I. Programa de promoción de la salud en una comunidad nativa de Alaska: un caso de planificación estratégica y colaboración comunitaria. Salud Uninorte.2013 Nov; 29 (3):.418-419.

51. Castro A. La nutrición como ruptura cultural: la experiencia de los adultos con diabetes mellitus tipo 2 . Investigación en salud 2007 Abr; 9(1): 26-33

52. Velásquez C, Yapes J, Ariza K, Isaza P, Isaza A, Gaviria A, et al. Estimación de la calidad de vida relacionada con la salud y medidas de utilidad en una población de pacientes colombianos con lupus eritematoso sistémico. ColombReumatol. 2013 Dic; 20(4): 183-194.
53. Ferro R, García M, Vives M. Un análisis de la adherencia al tratamiento en fisioterapia. Fisioterapia, 2004; 26 (6): 333-339.

54. Ariza K, Isaza P, Gaviria AM, Quinceno JM, Vinaccia S, Alvarán L, et al. Calidad de vida relacionada con la salud, factores psicológicos y fisiopatológicos en pacientes con diagnóstico de Lupus Eritematoso Sistémico - LES Terapia Psicológica. Terapia de psicología, 2010 Jul; 28(1):27-36.

55. Organización Mundial de la Salud. Centro de prensa, enfermedades no trasmisible [internet]. [Consultado 2015 Ene 17]. Disponible en: http://www.who.int/ mediacentre/factsheets/fs355/es/

56. Colombia. Ministerio de la Protección Social. Decreto 3518 de 2006. Por el cual se crea y reglamenta el Sistema de Vigilancia en Salud Pública y se dictan otras disposiciones. Diario Oficial, 46417(Oct. 10 2006)

57. Vinaccia S, Orozco L. Aspectos psicosociales asociados con la calidad de vida de personas con enfermedades crónicas Diversitas: Perspectivas en Psicología. 2005 Jun; 1(2): 125-137.

58. Colombia Ministerio de la Protección Social. Ley 1122 de enero 9 de 2007 . Por la cual se hacen algunas modificaciones en el Sistema General de Seguridad Social en Salud y se dictan otras disposiciones. Diario Oficial, 46506 (Ene.9 2007)

59. Polanco N, Soto M, Rodríguez F. Presentación clínicopatológica de la nefropatía lúpica: experiencia de un centro mexicano. Colomb Reumatol, 2013 Abr; 20(2):80-90.

60. Ramos R, Molina M. Nuevos modelos de gestión de asistencia integral en nefrología. Nefrología. Órgano Oficial de la Sociedad Española de Nefrología. 2013 Feb; 33(3): 301-7.

Para citar este artículo: Gaviria-García G, Aroca-Martínez G, Lastre-Amell G, Sierra-Llamas C. Láser de diodo; Atención interdisciplinar para una adecuada adherencia al tratamiento en pacientes con nefritis lúpica. Duazary. 2016 enero; 13 (1): 30 - 39 